\title{
Impact of Concomitant Thyroid Pathology on Preoperative WORKUP FOR PRIMARY HYPERPARATHYROIDISM
}

\author{
O. Heizmann ${ }^{1}$, C. T. Viehl ${ }^{1}$, R. Schmid ${ }^{1}$, J. Müller-Brand ${ }^{2}$, B. Müller ${ }^{3}$, D. Oertli ${ }^{1}$ \\ ${ }^{1}$ Allgemeinchirurgische Klinik, Departement Chirurgie, Universitätsspital Basel, Switzerland \\ ${ }^{2}$ Klinik und Institut für Nuklearmedizin, Universitätsspital Basel, Switzerland \\ ${ }^{3}$ Klinik für Endokrinologie, Diabetologie und klinische Ernährung, Departement Innere Medizin, Universitätsspital Basel, Switzerland
}

\begin{abstract}
Background: The former standard surgical treatment in patients with primary hyperparathyroidism (pHPT) has been bilateral cervical exploration. New localization techniques and the possibility of intraoperative measurement of intact parathormone (iPTH) permit a focused, minimally invasive parathyroidectomy (MIP). The introduction of MIP without complete neck exploration leads to the potential risk of missing thyroid pathology. The aim of the present study is to evaluate the value of MIP in respect to coexisting thyroid findings and their impact on preoperative workup for primary hyperparathyroidism.

Methods: This is a prospective study including 30 consecutive patients with pHPT (median age 65 years; 17 females, 13 males). In all patients preoperative localization was performed by ultrasonography and $99 \mathrm{~m}$ TcMIBI scintigraphy- Intraoperative iPTH monitoring was routinely done.

Results: Ten patients (33\%) had a concurrent thyroid finding requiring additional thyroid surgery, and two patients $(7 \%)$ with negative localization results underwent bilateral neck exploration. Therefore, MIP was attempted in $18(60 \%)$ patients. The conversion rate to a four gland exploration was $6 \%(1 / 18)$. The sensitivities of $99 \mathrm{~m}$ Tc-MIBI scanning and ultrasonography were $83.3 \%$ and $76.6 \%$, respectively. The respective accuracy rates were $83.3 \%$ and $76.6 \%$. Of note, the combination of the two modalities did not improve the sensitivity and accuracy in our patient population. During a median follow-up of 40 months, none of the patients developed persistent or recurrent hypocalcaemia, resulting in a $100 \%$ cure rate.

Conclusion: Coexisting thyroid pathology is relatively frequent in patients with $\mathrm{pHPT}$ in our region. Among patients having pHPT without any thyroid pathology, the adenoma localization is correct with either ultrasonography or ${ }^{99} \mathrm{~m}$ Tc-MIBI scintigraphy in the majority of cases. MIP with iPTH monitoring are highly successful in this group of patients and this operative technique should be the method of choice.
\end{abstract}

Key words: Primary Hyperparathyroidism, parathyroid imaging, parathormone monitoring, surgery, thyroid pathology

\section{INTRODUCTION}

Parathyroidectomy is the accepted curative option for hyperparathyroidism (HPT). Definite preoperative localization of parathyroid adenomas and advances in surgical techniques in combination with intraoperative measurement of intact parathormone (iPTH) have made minimally invasive parathyroidectomy (MIP) an acceptable surgical approach for patients with primary hyperparathyroidism (pHPT) [1-4]. Therefore, MIP has become a widely-accepted alternative to the standard four gland exploration for patients with pHPT. Over the past ten years, several different approaches of MIP have been described. These include total endoscopic parathyroidectomy, video-assisted techniques, radio-guided explorations and focused parathyroidectomy using mini-incisions either by a central or lateral approach directly over the adenoma [3, 5-8]. All these techniques have considerable advantages and disadvantages. MIP has been reported to reach success rates over $90 \%$ and to be associated with a low complication rate. Common to all MIP procedures is the abandonment of routine bilateral neck exploration in patients undergoing parathyroidectomy. These minimal invasive procedures are mostly based on an unequivocal preoperative localization of parathyroid adenomas on $99 \mathrm{~m}$-Technetium-Methoxyisobutylisonitril ( $99 \mathrm{~m}$ 'Tc-MIBI) scintigraphy. With MIP the surgeon may not be able to examine the entire thyroid gland for associated pathology. The rate of incidentally detected thyroid pathology in patients with pHPT has been reported to range from 17\%-84\% [913]. The frequency of malignant thyroid lesions in population with $\mathrm{PHPT}$ ranges from $2 \%$ to $12 \%[9,11$, $14,15]$.

To improve the investigative success for most thyroid pathologies ultrasonography should be used in addition to $99 \mathrm{~m}$ Tc-MIBI scan [16-18]. Ultrasound enables to screen the thyroid gland for pathologies while localizing the parathyroid adenoma at the same time. However, early diagnosis and simultaneous surgical treatment especially for thyroid cancer continues to be a central part of treatment. Delayed diagnosis can result in more difficulties at the time of second neck exploration, and in an increased complication rate. 
The aim of the present study is to evaluate the value of focused open MIP in respect to coexisting thyroid findings and their impact on preoperative workup for primary hyperparathyroidism.

\section{Patients And Methods}

From April 2003 to November 2006 a total of 46 parathyroidectomies were performed at our institution. Sixteen had secondary and tertiary HPT and were excluded from the study. The study was approved by the of the Basel University hospital ethical committee and each patient gave informed consent to participate the study.

Therefore, 30 patients with pHPT were included in this prospective, non-randomized study. There were 17 female and 13 male patients with a median age of 65 years (range 34-77). Clinical symptoms were fatigue and depression in $5 / 30$ patients, concurrent hypertension in $14 / 30$, nephrolithiasis in $6 / 30$, bone disease (elevated alkaline phosphatase) in $16 / 30$, pain in the extremities in $3 / 30$ and peptic ulcer in $2 / 30$. Five patients were asymptomatic.

The choice of operative approach was influenced by the localization results. All patients received preoperative $99 \mathrm{~m}$ Tc-MIBI scanning and ultrasonography for localization of diseased parathyroid. The thyroid was additionally assessed during ultrasonography.

Exclusion criteria for MIP contained the presence of concomitant thyroid disease with necessity for surgical treatment, a suspicion of multiglandular disease, and negative or not conclusive preoperative localization. Patients with concomitant thyroid pathology did not routinely undergo a fine needle aspiration (FNA) as they unequivocally needed surgery for their hyperparathyroidism.

Patients qualifying for MIP received an offer to choose between local and general anesthesia. The skin incision was limited to a length of $2.5 \mathrm{~cm}$ preferably in a natural skin crease. A focused MIP was then performed based on preoperative localization results. Surgery in patients with coexisting thyroid pathology was always performed under general anesthesia and exploration was started on the side where pathologies have been found in imaging studies. In patients without conclusive preoperative localization regarding parathyroid adenoma the neck exploration was started on the side of thyroid pathology with the intention to limit surgery to unilateral exploration.

Since 1990 a commercially available immuno-chemiluminometric assay (IMMULITE Turbo intact PTH, Diagnostic Products Corporation, Los Angeles USA) was applied for quantification of iPTH. This 15 minute quick assay uses two polyclonal antibodies against intact PTH, one coated on a polystyrene bead and another conjugated to alkaline phosphatase. Results were usually transmitted within 30 minutes by phone to the operating theatre. PTH levels were monitored at baseline ( 5 minutes before skin incision), and 10 minutes after parathyroidectomy. An decrease of iPTH levels of more than $78 \%$ from baseline 10 minutes after removal of the parathyroid gland is equivalent to successful resection of enlarged glands [19]. We assessed the serum calcium levels on the first postoperative day and documented potential surgical and metabolic complications.

\section{RESULTS}

This study includes 30 patients treated for $\mathrm{pHPT}$. Ten patients $(33 \%)$ had a concurrent thyroid finding (Table 1) requiring additional thyroid surgery. Four patients of those with thyroid and parathyroid pathology on the same side received unilateral exploration. Two patients $(7 \%)$ with negative localization results underwent bilateral neck exploration.

Eighteen patients $(60 \%)$ met the criteria for open MIP based on successful localization of a solitary enlarged gland with $99 \mathrm{~m}$ Tc-MIBI scintigraphy and ultrasonography. One out of these 18 got no conclusive results using this approach. She was additionally studied using magnetic resonance imaging (MRI) that eventually revealed a single enlarged parathyroid adenoma dorsal from the left thyroid lobe.

The sensitivities of $99 \mathrm{~m}$ Tc-MIBI scanning and of ultrasonography in this study were $83.3 \%$ and $76.6 \%$, respectively (Table 2). As expected the sensitivities of both localization modalities were higher and lower in patients without and with thyroid pathology, respectively (Table 3 and 4). Of note, the combination of the two modalities did not improve the sensitivity in our patient population. The exact intraoperative loca-

Table 1. Concomitant thyroid pathology.

\begin{tabular}{lc}
\hline Pathology & n \\
\hline Multinodular goiter & 4 \\
Uninodular goiter & 2 \\
Hashimoto thyroiditis & 1 \\
Thyroid adenoma & 1 \\
Oncocytic neoplasia & 1 \\
Papillary thyroid carcinoma pT3,pN1 $(2 / 20), \mathrm{G} 2$ & 1 \\
Total & 10 \\
\hline
\end{tabular}

Table 2. Reliability of Sesta-MIBI and Ultrsonography for Location of Parathyroid Adenomas.

\begin{tabular}{|c|c|c|c|c|c|c|c|c|}
\hline Modality & $\mathrm{n}$ & True positive & False positive & True negative & False negative & $\begin{array}{c}\text { Sensitivity } \\
(\%)\end{array}$ & $\begin{array}{c}\text { Specificity } \\
(\%)\end{array}$ & $\begin{array}{c}\text { Accuracy } \\
(\%)\end{array}$ \\
\hline Sestamibi & 30 & 25 & 0 & 0 & 5 & 83.3 & - & 83.3 \\
\hline Ultrasound & 30 & 22 & 0 & 0 & 7 & 76.6 & - & 76.6 \\
\hline Combined & 30 & 25 & 0 & 0 & 5 & 83.3 & - & 83.3 \\
\hline
\end{tabular}


Table 3. Reliability of Sesta-MIBI and Ultrsonography for Location of Parathyroid Adenomas with concomitant Thyroid Pathology.

\begin{tabular}{|c|c|c|c|c|c|c|c|c|}
\hline Modality & $\mathrm{n}$ & True positive & False positive & True negative & False negative & $\begin{array}{c}\text { Sensitivity } \\
(\%)\end{array}$ & $\begin{array}{c}\text { Specificity } \\
(\%)\end{array}$ & $\begin{array}{c}\text { Accuracy } \\
(\%)\end{array}$ \\
\hline Sestamibi & 12 & 8 & 0 & 0 & 4 & 66.6 & - & 66.6 \\
\hline Ultrasound & 12 & 7 & 0 & 0 & 5 & 58.3 & - & 58.3 \\
\hline Combined & 12 & 8 & 0 & 0 & 4 & 66.6 & - & 66.6 \\
\hline
\end{tabular}

Table 4. Reliability of Sesta-MIBI and Ultrsonography for Location of Parathyroid Adenomas without concomitant Thyroid Pathology.

\begin{tabular}{lcccccccc}
\hline Modality & $\mathrm{n}$ & True positive & False positive & True negative & False negative & $\begin{array}{c}\text { Sensitivity } \\
(\%)\end{array}$ & $\begin{array}{c}\text { Specificity } \\
(\%)\end{array}$ & $\begin{array}{c}\text { Accuracy } \\
(\%)\end{array}$ \\
\hline Sestamibi & 18 & 17 & 0 & 0 & 1 & 94.4 & - & 94.4 \\
Ultrasound & 18 & 16 & 0 & 0 & 2 & 88.8 & - & 88.8 \\
Combined & 18 & 17 & 0 & 0 & 1 & 94.4 & - & 94.4 \\
\hline
\end{tabular}

tion of the adenomas was different in six and seven than predicted by $99 \mathrm{~m}$ Tc-MIBI scan and by ultrasound, respectively. This results in a gland-specific (exact anatomic location) accuracy rate of $83.3 \%$ and $76.6 \%$, respectively. One patient without conclusive preoperative localization had two glands removed. Histological examination revealed a double adenoma situation and a rate of multiglandular disease rate of $3 \%$.

MIP was successfully carried out in $17 / 18$ patients $(94 \%)$. Because of an ectopically located (paraesophageal) right upper gland, which has originally been attributed to a right lower parathyroid by imaging, one patient $(1 / 18)$ needed conversion to the conventional unilateral approach (i.e. a conversion rate of $6 \%$ ). Three patients opted for MIP under local anesthesia, which was successfully carried out in all three cases.

The median preoperative levels for serum calcium and PTH was $2.89 \mathrm{mmol} / 1$ (range $2.71-3.7 \mathrm{mmol} / \mathrm{l}$; normal values are $2.10-2.65 \mathrm{mmol} / \mathrm{l})$ and $250 \mathrm{pg} / \mathrm{ml}$ (range 102 - $1892 \mathrm{pg} / \mathrm{ml}$; normal values $10-73 \mathrm{pg} / \mathrm{ml}$ ), respectively.

During the initial postoperative course twenty-six patients were normocalcaemic, four were subclinical hypocalcaemic and one patient (after conventional four gland exploration) developed hoarseness due to transient recurrent laryngeal nerve palsy. This resolved within two weeks postoperatively. Therefore no permanent postoperative complication occurred. The median postoperative PTH level (10 minutes after adenoma removal) was $33 \mathrm{pg} / \mathrm{ml}$ (range, $17-80 \mathrm{pg} / \mathrm{ml}$ ). The median postoperative calcium level 24 hours postoperatively was $2.24 \mathrm{mmol} / 1$ (range, $1.94-2.59 \mathrm{mmol} / \mathrm{l}$ ).

All 17 patients after MIP procedure and 9 patients after conventional four gland exploration (26 out of 30 ) were discharged 24 hours postoperatively, whereas the remaining four patients with hypocalcaemia needed longer observation and were discharged 48 hours after surgery.

Calcium levels at least 1 month after surgery were available in all 30 patients $(100 \%)$ at a median follow- up time of 40 (range 21-63) months. During follow-up evaluation no patient developed persistent or recurrent hyperparathyroidism. Histological examination of all removed glands was confirmed benign parathyroid tissue changes. Thus, with the use of iPTH monitoring, MIP and conventional neck exploration resulted in a $100 \%$ success rate.

\section{DisCUSSION}

Today, minimally invasive, targeted parathyroidectomy has achieved wide acceptance among endocrine surgeons. The present investigation provides evidence that MIP is feasible in about two thirds of patients with $\mathrm{pHPT}$ in an endemic goiter region. However, the $33 \%$ proportion of patients with additional thyroid findings is quite high. Nevertheless, a 100\% cure rate could be achieved by the selective use of MIP combined with the conventional neck exploration for patients who were not suitable for MIP at our institution.

Surgery is the accepted curative option for symptomatic and asymptomatic hyperparathyroidism, respectively. The introduction and refinement of parathyroid imaging was a pre-condition for the development of MIP techniques. Several non-invasive preoperative localization methods are available, including $99 \mathrm{~m}$ Tc-MIBI scintigraphy, ultrasonography, CT, MRI and thalliumtechnetium-pertechnetate subtraction scanning. Today, the most common procedures for preoperative localization are ${ }^{99} \mathrm{~m}$ 'Tc-MIBI scintigraphy and ultrasonography. A meta-analysis of sensitivity and specificity of 99m Tc-MIBI scanning provided rates of $90,7 \%$ and $98,8 \%$, respectively, and suggested that more than $80 \%$ of the patients with pHPT would be candidates for MIP [20]. Of note, these data originate from regions where goitrous changes are not endemic. In our institution, situated in a region where goiter is endemic, 33\% of patients presented with additional thyroid pathologies. Still, this leaves two thirds of patients being eligible for MIP. 
${ }^{99 \mathrm{~m}}$ Tc-MIBI scanning is helpful in evaluation of hyperfunctional parathyroid tissue in the neck and in the mediastinum as well. With ultrasound only the neck region can reliably be scanned. A major limitation of $99 \mathrm{~m}$ Tc-MIBI scans is, however, related to multiglandular parathyroid disease [21] and to the concomitant thyroid pathology that can mimic parathyroid adenomas resulting in false-positive results. The results of present study are also supporting this influence of coexisting thyroid pathology on preoperative work up. This limitation can be managed by combining SestaMIBI scanning with neck ultrasound which usually distinguishes between thyroid nodules and enlarged parathyroid glands $[22,23]$. The ultrasonography is effective, inexpensive and shows true-positive rates approaching 90\% when combined with $99 \mathrm{~m}$ Tc-MIBI [24]. CT and MRI both provide cross-sectional imaging that can occasionally be useful for localization of enlarged parathyroid glands that are ectopically situated retropharyngeal and retroesophageal within the neck and the mediastinum as shown in this study. CT is less expensive, and many studies have shown that these two techniques have an equal sensitivity. The innovative SPECT /CT modality enables the combined scanning with image fusion resulting in a more precise anatomic localization of the tracer activity.

The high success of MIP has been confirmed by evidence of cure without complications in our study that are at least as good as those achieved by conventional bilateral neck exploration $[3,25,26]$. The potential advantages of MIP include the possible use of local anesthesia, reduced operating times of up to $50 \%$, a lower incidence of hypocalcaemia, reduction of hospital stay and reduction by nearly one half in total hospital charges [3]. In line with this, in the present study no hypocalcaemia was noted in patients undergoing MIP. Furthermore, after MIP all patients were discharged 24 hours postoperatively. Based on these benefits we currently prefer MIP as described by Udelsman et al. [27].

The rapid PTH assay for intraoperative hormone monitoring has an integral role in the intraoperative decision process during MIP. According to most assays, a $50 \%$ reduction in the quick iPTH value from the baseline level is used as an indication that surgery has been successful and this is predictive of cure in $96 \%$ of cases [28]. Based on a recently published prospective validation study we advocate an iPTH decrease to below $22 \%$ from the baseline level at $10 \mathrm{~min}$ utes after parathyroidectomy to be most accurate for complete removal of all hyperplastic parathyroid tissue [19]. We are convinced that such a stringent decrease of iPTH is imperative to distinguish between singleand multiglandular disease. With a $50 \%$ reduction rate at 10 minutes only, as formerly proposed by Irvin [29], we could potentially have missed multiglandular disease.

In conclusion, coexisting thyroid findings are relatively frequent in patients with $\mathrm{pHPT}$ and substantially influence the preoperative workup in our region. U1trasonography helped identifying concomitant thyroid pathologies in a large subset of our patient population. We therefore recommend the routine use of ultrasonography in combination with ${ }^{99} \mathrm{~m}$ Tc-MIBI scinti- graphy in regions where goiter is endemic. However, among patients having $\mathrm{pHPT}$ without any thyroid pathology, the adenoma localization is correct with either ultrasonography or $99 \mathrm{~m}$ Tc-MIBI scintigraphy in the majority of cases. MIP with iPTH monitoring and is highly successful in this group of patients and the operative technique is regarded to be the method of choice. The imperative condition for a successful MIP is an accurate preoperative localization test (i.e. ${ }^{99 \mathrm{~m} T c-}$ MIBI) combined with the evaluation for coexisting thyroid abnormalities (i.e. cervical ultrasound), particularly with respect to one-stage surgery for both pathologies.

\section{REFERENCES}

1. Howe JR. Minimally invasive parathyroid surgery. Surg Clin North Am. 2000; 80(5): 1399-1426.

2. Miccoli P. Minimally invasive surgery for thyroid and parathyroid diseases. Surg Endosc. 2002; 16(1): 3-6.

3. Udelsman R. Six hundred fifty-six consecutive explorations for primary hyperparathyroidism. Ann Surg. 2002; 235(5): 665-670.

4. Udelsman R, Donovan PI. Open minimally invasive parathyroid surgery. World J Surg. 2004; 28(12): 12241226.

5. Agarwal G, Barraclough BH, Robinson BG, et al. Minimally invasive parathyroidectomy using the 'focused' lateral approach. I. Results of the first 100 consecutive cases. ANZ J Surg. 2002; 72(2): 100-104.

6. Gagner M, Inabnet WB, III. Endoscopic thyroidectomy for solitary thyroid nodules. Thyroid. 2001; 11(2): 161163.

7. Sackett WR, Barraclough B, Reeve TS, et al. Worldwide trends in the surgical treatment of primary hyperparathyroidism in the era of minimally invasive parathyroidectomy. Arch Surg. 2002; 137(9): 1055-1059.

8. Yeung GH, Ng JW. The technique of endoscopic exploration for parathyroid adenoma of the neck. Aust N Z J Surg. 1998; 68(2): 147-150.

9. Bentrem DJ, Angelos P, Talamonti MS, et al. Is preoperative investigation of the thyroid justified in patients undergoing parathyroidectomy for hyperparathyroidism? Thyroid. 2002; 12(12): 1109-1112.

10. Lever EG, Refetoff S, Straus FH, et al. Coexisting thyroid and parathyroid disease--are they related? Surgery. 1983; 94(6): 893-900.

11. Prinz RA, Barbato AL, Braithwaite SS, et al. Simultaneous primary hyperparathyroidism and nodular thyroid disease. Surgery. 1982; 92(3): 454-458.

12. Strichartz SD, Giuliano AE. The operative management of coexisting thyroid and parathyroid disease. Arch.Surg. 1990;125(10)1327-1331.

13. Monroe DP, Edeiken-Monroe BS, Lee JE, et al. Impact of preoperative thyroid ultrasonography on the surgical management of primary hyperparathyroidism. Br J Surg. 2008; 95(8): 957-960.

14. Attie JN, Vardhan R. Association of hyperparathyroidism with nonmedullary thyroid carcinoma: review of 31 cases. Head Neck. 1993; 15(1): 20-23.

15. Burmeister LA, Sandberg M, Carty SE, et al. Thyroid carcinoma found at parathyroidectomy: association with primary, secondary, and tertiary hyperparathyroidism. Cancer. 1997; 79(8): 1611-1616.

16. Ammori BJ, Madan M, Gopichandran TD, et al. Ultrasound-guided unilateral neck exploration for sporadic primary hyperparathyroidism: is it worthwhile? Ann R Coll Surg Engl. 1998; 80(6): 433-437. 
17. Haciyanli M, Lal G, Morita E, et al. Accuracy of preoperative localization studies and intraoperative parathyroid hormone assay in patients with primary hyperparathyroidism and double adenoma. J Am Coll Surg. 2003; 197(5): 739-746.

18. Lane MJ, Desser TS, Weigel RJ, et al. Use of color and power Doppler sonography to identify feeding arteries associated with parathyroid adenomas. AJR Am J Roentgenol. 1998; 171(3): 819-823.

19. Beck TM, Huber PR, Oertli D. Intraoperative parathormone measurement in patients with primary hyperparathyroidism: a prospective clinical study. Swiss Med Wkly. 2003; 133(13-14): 206-209.

20. Denham DW, Norman J. Cost-effectiveness of preoperative sestamibi scan for primary hyperparathyroidism is dependent solely upon the surgeon's choice of operative procedure. J Am Coll Surg. 1998; 186(3): 293-305.

21. Blanco I, Carril JM, Banzo I, et al. Double-phase Tc-99m sestamibi scintigraphy in the preoperative location of lesions causing hyperparathyroidism. Clin Nucl Med. 1998; 23(5): 291-297.

22. Casara D, Rubello D, Cauzzo C, et al. ${ }^{99 \mathrm{~m} T \mathrm{Tc}-\mathrm{MIBI} \text { radio- }}$ guided minimally invasive parathyroidectomy: experience with patients with normal thyroids and nodular goiters. Thyroid. 2002; 12(1): 53-61.

23. Milas M, Mensah A, Alghoul M, et al. The impact of office neck ultrasonography on reducing unnecessary thyroid surgery in patients undergoing parathyroidectomy. Thyroid. 2005; 15(9): 1055-1059.

24. Geatti O, Shapiro B, Orsolon PG, et al. Localization of parathyroid enlargement: experience with technetium$99 \mathrm{~m}$ methoxyisobutylisonitrile and thallium-201 scintigraphy, ultrasonography and computed tomography. Eur J Nucl Med. 1994; $21(1): 17-22$.
25. Bergenfelz A, Kanngiesser V, Zielke A, et al. Conventional bilateral cervical exploration versus open minimally invasive parathyroidectomy under local anaesthesia for primary hyperparathyroidism. Br J Surg. 2005; 92(2):190197.

26. Chen H, Sokoll LJ, Udelsman R. Outpatient minimally invasive parathyroidectomy: a combination of sestamibiSPECT localization, cervical block anesthesia, and intraoperative parathyroid hormone assay. Surgery. 1999; 126(6): 1016-1021.

27. Udelsman R, Donovan PI, Sokoll LJ. One hundred consecutive minimally invasive parathyroid explorations. Ann Surg. 2000; 232(3): 331-339.

28. Garner SC, Leight GS, Jr. Initial experience with intraoperative PTH determinations in the surgical management of 130 consecutive cases of primary hyperparathyroidism. Surgery. 1999; 126(6): 1132-1137.

29. Irvin GL, III, Sfakianakis G, Yeung L, et al. Ambulatory parathyroidectomy for primary hyperparathyroidism. Arch Surg. 1996; 131(10): 1074-1078.

Received: August 4, 2008 / Accepted: October 30, 2008

Address for correspondence:

Dr. med. Oleg Heizmann

Allgemeinchirurgische Klinik

Universitätsspital Basel

Spitalstrasse 21

CH-4031 Basel

Switzerland

Phone: $004161 / 2652525$

Fax: $\quad 004161 / 2657250$

E-mail: oheizmann@uhbs.ch 